positive and symptomatic men. While a prevalence of $9 \%$ would prompt discussion of screening, more needs to be known about the natural history of MG in MSM, given the high levels of antimicrobial resistance and risk associated with quinolones. MG was uncommon in the pharynx of MSM (1\%), suggesting this site is not a significant source of transmission and testing is not indicated.

Disclosure No significant relationships.

\section{P526 HIGH PREVALENCE AND INCIDENCE RATE OF RECTAL CHLAMYDIA AND GONORRHOEA INFECTION AMONG MEN WHO HAVE SEX WITH MEN IN TOKYO}

${ }^{1}$ Daisuke Mizushima*, ${ }^{2}$ Misao Takano, ${ }^{1}$ Haruka Uemura, ${ }^{1}$ Yasuaki Yanagawa, ${ }^{1}$ Takahiro Aoki, ${ }^{1}$ Koji Watanabe, ${ }^{1}$ Shinichi Oka. ${ }^{1}$ National Center for Global Health and Medicine, AIDS Clinical Center, Tokyo, Japan; ${ }^{2}$ National Center for Global Health and Medicine, Tokyo, Japan

\subsection{6/sextrans-2019-sti.604}

Background Rectal Chlamydia trachomatis (CT) and Neisseria gonorrhoeae (NG) infection have been neglected and epidemiological data is not available in Japan. Thus, we evaluated prevalence and incidence of rectal CT and NG in non HIVinfected men who have sex with men (MSM) cohort, Sexual Health Clinic (SHC) established in National Center for Global Health and Medicine (NCGM), in Tokyo in January 2017.

Methods Non HIV-infected MSM with aged 16 years old and over were included in SHC. Prevalence of rectal CT/GC infection in SHC were compared with those of an existing HIVinfected MSM cohort in NCGM. The participants of SHC were examined HIV infection and rectal and pharyngeal CT/ NG every 3 months. Urethral CT/NG infection were evaluated at physician's discretion. Incidence rate of HIV, CT and NG were evaluated at the time of December 2018.

Results 502 MSM had been included into SHC by December 2018 and 13 were diagnosed with HIV infection at the enrollment and excluded. $561 \mathrm{HIV}$-infected MSM were evaluated rectal CT/NG infection cross-sectionally. Between the two cohorts, mean age was 33.6 and 46.4 years old $(p<0.001)$. Prevalence of rectal CT or NG infection were $18.4 \%$ and $16.8 \%(p=0.483)$. Prevalence of rectal CT was $16.4 \%$ and $15.5 \%(p=0.707)$ and prevalence of NG was $4.1 \%$ and $2.3 \%$ $(\mathrm{p}=0.101)$. Of the 489 subjects in SHC, 326 were followed at least twice, with 291.8 person-years during the study period. The incidence of HIV, rectal CT and NG infection were 3.8, 18.8, 4.9/100 person-year. The incidence of pharyngeal CT and NG infection were 1.5 and 8.3/100 personyear. $33.8 \%$ of the incidental cases were recurrent infection. In this study, HIV seroconversion was not associated with rectal CT or NG infection at the enrollment $(p=0.438)$.

Conclusion The prevalence and incidence of rectal CT and NG infection are high among MSM in Tokyo, which requires urgent countermeasures.

Disclosure No significant relationships.

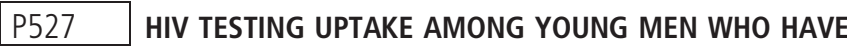 SEX WITH MEN IN MYANMAR: SELF-EFFICACY AND HIV TESTING BEHAVIOR}

Minh Pham. Burnet Institute, Public Health, Melbourne, Australia

10.1136/sextrans-2019-sti.605
Background Men who have sex with men (MSM) are disproportionally affected by the HIV epidemic. HIV testing coverage among MSM, particularly in low-resource settings, remains low. Self-efficacy is an important individual psychosocial factor associated with access to health care and health outcomes but the association between self-efficacy and HIV testing uptake among key populations is poorly understood.

Methods A cross sectional study was conducted with 585 MSM aged 18-24 years recruited by 33 seed respondents from six urban areas in Myanmar via respondent-driven sampling (RDS). RDS analyses were performed to provide population estimates of HIV testing coverage. Sensitivity analysis was performed to assess potential bias due to inclusion of seed and HIV positive respondents on final population estimates. Multinomial logistic regression was use to examine the relationship between self-efficacy and HIV testing uptake.

Results More than a third (34.5\%) had never been tested for HIV (never tester), $27.5 \%$ and $38.0 \%$ had their most recent HIV test more than three months (non-recent tester) and within the past three months (recent tester), from the time of interview, respectively. Sensitivity analysis showed similar point estimates excluding seed or HIV positiverespondents (Table1). Results of multinomial logistic regression analyses show that YMSM who reported high self-efficacy (adjusted Relative Risk Ratio $[A R R]=7.35,95 \%$ CI: 2.29-23.5) and moderate selfefficacy $(\mathrm{ARR}=8.61,95 \% \mathrm{CI}: 3.09-24.0)$ are more likely to report having tested for HIV in the past three months compared to their counterparts who reported low self-efficacy after adjusting for potential confounders.

Conclusion HIV testing coverage among YMSM is suboptimal and there is a positive association between self-efficacy and HIV testing uptake among YMSM. Further research is needed to examine the direction of this association to inform future public health interventions targeting YMSM in Myanmar. Disclosure No significant relationships.

\section{P528 RATES OF PRIMARY AND SECONDARY SYPHILIS AMONG MEN WHO HAVE SEX WITH MEN BY HIV STATUS - 24 STATES, 2011-2015}

Jeremy Grey*, Elizabeth Torrone, Hillard Weinstock. US Centers for Disease Control and Prevention, Division of STD Prevention, Atlanta, USA

\subsection{6/sextrans-2019-sti.606}

Background During 2011-2015, approximately half (48\% to $53 \%)$ of reported primary and secondary (P\&S) syphilis cases occurred among men who have sex with men (MSM) with diagnosed HIV. However, comparing rates of reported P\&S syphilis by HIV status among MSM requires MSM population denominators stratified by HIV status.

Methods We used previously published state-level estimates of the MSM population and publicly available data on diagnosed HIV infection among MSM to determine population denominators of MSM living with or without diagnosed HIV infection. We then examined rates of reported P\&S syphilis per 100,000 MSM by HIV status using national case-based surveillance data in 24 states with $\geq 70 \%$ complete reporting of sex of sex partners and HIV status among syphilis cases during 2011-2015.

Results During 2011-2015, rates of reported P\&S syphilis among MSM in the 24 states included in the analysis increased $45 \%$ (188.7 to 272.8 per 100,000). Concurrently, 
rates among HIV-negative and HIV-unknown MSM, combined, increased 64\% (83.0 to 136.5 per 100,000$)$, while rates among MSM with diagnosed HIV increased 17\% during 2011-2014 (1,061.5 to $1,237.3$ per 100,000$)$ before decreasing $6 \%$ to $1,229.5$ in 2015 . Rate ratios comparing reported P\&S syphilis rates among MSM living with diagnosed HIV to HIV-negative and HIV-unknown MSM decreased annually during this period from 12.8 to 9.0 .

Conclusion During the most recent five-year period for which data are available, rates of reported P\&S syphilis increased among MSM diagnosed with HIV, as well as MSM not diagnosed with HIV. Although rates are higher among MSM diagnosed with HIV, larger relative increases in rates among MSM not diagnosed with HIV and subsequent declining rate ratios indicated that differences between MSM with diagnosed HIV and HIV-negative or HIV-unknown MSM diminished over time.

Disclosure No significant relationships.

\section{P529 EARLY SEXUAL DEBUT AND NON-CONSENSUAL SEX AMONG CHINESE MEN WHO HAVE SEX WITH MEN: A MULTI-CITY CROSS-SECTIONAL STUDY}

${ }^{1}$ Weiming Tang*, ${ }^{1}$ Yehua Wang ${ }^{1}$ Jason Ong, ${ }^{1}$ Dan Wu, ${ }^{2} \mathrm{M}$ Kumi Smith, ${ }^{3} \mathrm{H}$ nongyun $\mathrm{Fu}$ 'Wenting Huang, 'Chongyi Wei, 'Joseph Tucker. 'UNC Project-China, Guangzhou, China; ${ }^{2}$ University of Minnesota Twin Cities, Epidemiology and Community Health, Minneapolis, USA; ${ }^{3}$ Eastern Virginia Medical School, Norfolk, USA; ${ }^{4}$ Rutgers, USA

\subsection{6/sextrans-2019-sti.607}

Background Early sexual debut and non-consensual sex among men who have sex with men (MSM) may contribute to adult high-risk behaviors and STI transmission. Most knowledge about early MSM sex comes from high-income countries. This study examined the prevalence and factors associated with the early sexual debut and non-consensual sex at sexual debut among Chinese MSM.

Methods An online cross-sectional study was conducted among MSM who were born as a male, $\geq 16$ years-old, and ever engaged in anal sex with a man in China in 2016. Participants answered questions regarding sociodemographics, condomless sex, and HIV testing history. Early sexual debut was defined as having anal sex before 18 . Non-consensual sex was defined as "where a person do sexual things to you that you did not want them to do." Multivariable logistic regression was used to identify factors associated with the early sexual debut and non-consensual sex.

Results Overall, 2105 men completed the survey. Among them, $85.9 \%$ were never married, and $35.4 \%$ had high school or less education. The mean age of sexual debut was 20.82 $(S D=5.30)$ years. About one-fifth $(20.1 \%, 424 / 2105)$ of men experienced early sexual debut, and 4.9\% (104/2105) experience non-consensual sex at sexual debut. Results from multivariable logistic regression models showed that early sexual debut was associated with having more male partners in the last three months (adjusted OR 1.08 [95\% CI 1.04-1.13]) and condomless sex in the last three months (1.49 [1.18-1.89]). MSM whose sexual debut was non-consensual were more likely to have tested for HIV (1.56 [1.01-2.42]), have had recent condomless sex (1.70 [1.13-2.56]), and to experience early sexual debut (2.54 [1.65-3.89]).

Conclusion Many Chinese MSM experience early sexual debut and non-consensual sex at sexual debut. Given the limited coverage of sexual health education in China, this has implications for expanding education and research about sexuality among youth.

Disclosure No significant relationships.

\section{P530 ADDRESSING UNDERSERVED MEN WHO HAVE SEX WITH MEN (MSM): ADVANCING THE SEXUAL HEALTH APPROACH FOR MSM IN VANCOUVER, CANADA}

${ }^{1}$ Tessa Lawson Tattersall, ${ }^{1}$ Nathan Lachowsky*, ${ }^{2}$ Mark Hull. ${ }^{1}$ University of Victoria, School of Public Health and Social Policy, Victoria, Canada; ${ }^{2}$ British Columbia Centre for Excellence in HIVIAIDS, Vancouver, Canada

\subsection{6/sextrans-2019-sti.608}

Background Men who have sex with men (MSM) are disproportionately overrepresented in the resurgence of sexually transmitted and blood-borne infections (STBBI) indicating a need to understand how access to sexual health services can be adapted for the improvement of the health and well-being of MSM. Our objective was to contextualize the access to and provision of MSM sexual health care with a syndemic lens. Accordingly, this study employs qualitative understanding of access to sexual health care, with particular focus on preexposure prophylaxis, for MSM in Vancouver.

Methods We conducted five focus groups from 12/2016-07/ 2017 with sexual health service providers serving MSM and members from the MSM community who have accessed sexual health care. Focus groups were audio recorded and transcribed verbatim. We iteratively analyzed data through a constant comparative technique to identify the accessibility of sexual health care for MSM in Vancouver with inference to syndemic effect. Results We identified service provider and MSM community attitudes and the structural concerns impacting access to sexual health care for MSM in Vancouver, Canada. Access to sexual health care assembled into three themes: (1) Perceptions and categorization of underserved; (2) Value of specialized MSM sexual health service; and (3) Capacity: reaching and referring. The first represents syndemics, reaching undiagnosed, representation and intersectionality, and specialized service providers as encouraging PrEP gatekeepers. The second combines MSM friendly environment, separation of sexual from other health care, and fear of association: community stigma and internalized shame. The third includes service promotion, capacity for HIV care, lack of counseling, and referral adequacy.

Conclusion MSM experience many biosocial interactions that negatively impact their access to sexual health care. Therefore, sexual health care would be more likely accessed if conditions were addressed together rather than separately. Findings offer social and structural-level interventions to address stigma and syndemic influence within MSM sexual health care delivery. Disclosure No significant relationships. 